Bulletin of Mathematical Biology (2001) 63, 1095-1124

doi: 10.1006/bulm.2001.0261

Available online at http://www.idealibrary.com on $\mathbf{D E} \mathbf{A}^{\infty}$

\title{
Critical Conditions for Phytoplankton Blooms
}

\author{
UTE EBERT*, MANUEL ARRAYÁS ${ }^{\dagger}$, NICO TEMME AND \\ BEN SOMMEIJER
}

CWI, P.O.Box 94079 ,

1090 GB Amsterdam,

The Netherlands

JEF HUISMAN

Aquatic Microbiology, Institute for Biodiversity and Ecosystem Dynamics, Universiteit van Amsterdam,

Nieuwe Achtergracht 127,

1018 WS Amsterdam,

The Netherlands

\begin{abstract}
We motivate and analyse a reaction-advection-diffusion model for the dynamics of a phytoplankton species. The reproductive rate of the phytoplankton is determined by the local light intensity. The light intensity decreases with depth due to absorption by water and phytoplankton. Phytoplankton is transported by turbulent diffusion in a water column of given depth. Furthermore, it might be sinking or buoyant depending on its specific density. Dimensional analysis allows the reduction of the full problem to a problem with four dimensionless parameters that is fully explored. We prove that the critical parameter regime for which a stationary phytoplankton bloom ceases to exist, can be analysed by a reduced linearized equation with particular boundary conditions. This problem is mapped exactly to a Bessel function problem, which is evaluated both numerically and by asymptotic expansions. A final transformation from dimensionless parameters back to laboratory parameters results in a complete set of predictions for the conditions that allow phytoplankton bloom development. Our results show that the conditions for phytoplankton bloom development can be captured by a critical depth, a compensation depth, and zero, one or two critical values of the vertical turbulent diffusion coefficient. These experimentally testable predictions take the form of similarity laws: every plankton-water-light-system characterized by the same dimensionless parameters will show the same dynamics.
\end{abstract}

(C) 2001 Society for Mathematical Biology

\footnotetext{
*Author to whom correspondence should be addressed. E-mail: ebert@cwi.nl

"Also at: Institute Lorento, Universiteit Leiden, The Netherlands and at Universidad Rey Juan Carlos, Madrid. Spain.

$0092-8240 / 01 / 061095+30 \quad \$ 35.00 / 0$

(C) 2001 Society for Mathematical Biology
} 


\section{INTRODUCTION}

Phytoplankton, the microscopically small plants that drift in the water column of lakes and oceans, provide the basis of nearly all food webs in aquatic ecosystems. Phytoplankton species depend on light for their metabolism. Hence, phytoplankton populations need to stay close to the surface, since light availability decreases with depth. On the other hand, many phytoplankton species are heavier than water. They have a tendency to sink. Sinking phytoplankton populations play an important role in climate regulation, as they can act as a carbon pump. By means of their photosynthetic carbon fixation, phytoplankton extract carbon dioxide from the atmosphere, and they can export a considerable part of this carbon by sinking downwards into the ocean interior (Falkowski et al., 1998; Arrigo et al., 1999; DiTullio et al., 2000). Here we will consider light as the factor limiting the growth rate of sinking phytoplankton populations. Thereby, we implicitly assume that all other resources, like nitrogen, phosphorus, and iron, are in ample supply. The sinking of phytoplankton can be compensated by turbulent mixing, or it can be stopped at the bottom of the water column. Hence, the question arises whether in an aquatic system with given depth, given rate of turbulent mixing, and given light intensity at the surface, a particular phytoplankton population will survive. Even for a single species, a full answer to this question is still missing.

If a phytoplankton population can survive in the long run, we will say that there is phytoplankton 'bloom development'. For well-mixed aquatic systems with homogeneous phytoplankton density, conditions for phytoplankton bloom development have been extensively investigated by means of integro-differential equation models (Sverdrup, 1953; Platt et al., 1991; Huisman and Weissing, 1994; Weissing and Huisman, 1994). Here, theory predicts that phytoplankton blooms can develop only if the depth of the well-mixed water column is less than a critical threshold value, generally known as the 'critical depth' in oceanography. If the depth of a mixed layer would exceed this critical depth, the average light intensity is too low to sustain a phytoplankton population. This theory for well-mixed systems is supported by extensive laboratory experiments (Huisman, 1999; Huisman et al., 1999a). Many, if not most, aquatic ecosystems are not well mixed, however. A few theoretical studies, using partial differential equation models, have investigated phytoplankton bloom development under incomplete mixing (Riley et al., 1949; Shigesada and Okubo, 1981; Totaro, 1989; Britton and Timm, 1993). In particular, recent numerical work considering neutrally buoyant phytoplankton led to the discovery of a critical threshold value for the vertical turbulent diffusion coefficient (Huisman et al., 1999b,c). If turbulent diffusion remains below this threshold value, populations of neutrally buoyant phytoplankton can outgrow the vertical mixing rates, and maintain a position in the upper well-lit part of the water column. Thus, the critical-depth theory applicable to well-mixed systems no longer holds if turbulent diffusion is sufficiently low. This finding was based on numerical simulations. A rigorous mathematical analysis of the conditions that 
allow phytoplankton bloom development under incomplete mixing is still lacking. In the present paper, we close this gap. Moreover, we generalize the analysis by including sinking or buoyancy of the phytoplankton. We analyse the long time survival conditions of a single phytoplankton species in the complete regime of possible physical parameters.

The paper is organized as follows: in Section 2, we introduce and motivate our model for one phytoplankton species. In Section 3, we perform a dimensional analysis that reduces the nine physical parameters of the problem to four dimensionless parameters called $A, B, C$ and the dimensionless water-column depth $L$. In Section 4, we prove that the phase boundaries that determine phytoplankton bloom development can be derived from a reduced linearized equation with homogeneous boundary conditions. This is the key for a simple numerical procedure, developed and used in Section 5, that characterizes the critical conditions for phytoplankton blooms in terms of a maximal dimensionless water-column depth $L^{*}=$ $L^{*}(A, B, C)$. In Section 6, we present our analytical results in terms of the dimensionless parameters. A key ingredient is the mapping of the linearized equation derived in Section 4 onto a Bessel function problem with particular boundary conditions. This allows for the identification of several limit cases and for asymptotic expansions about them. In Section 7, we return from the dimensionless variables $A$, $B$ and $C$ to physical variables like the diffusion constant $D$ or the light intensity $I_{\text {in }}$ at the surface, and we discuss under which conditions a (bounded) maximal water depth $H=H\left(D, I_{i n}\right)$ for a given phytoplankton species exists and how it can be derived from the previous analysis. Section 8 contains summary and conclusion.

\section{THE MODEL}

We here introduce our model for a single phytoplankton species. The phytoplankton population density is taken as a continuous quantity, and variations in the directions parallel to the water surface are neglected. Let $s$ denote the depth below the water surface, where $s$ runs from 0 at the surface to some $H>0$ at the bottom, and let $\omega(s, t)$ denote the phytoplankton population density at depth $s$ and time $t$. The changes in population density then take, in general, the form of a partial differential equation

$$
\frac{\partial}{\partial t} \omega(s, t)+\frac{\partial}{\partial s} j_{\omega}(s, t)=\mathcal{S}(\omega(s, t)) .
$$

This is the continuity equation for plankton, relating the local plankton density $\omega$ to the local plankton flux density $j_{\omega}$ and to the source term $\mathcal{S}(\omega(s, t))$ accounting for reproduction and death of the plankton. Plankton does not cross the air-waterand the water-ground-interface, therefore the boundary conditions at $s=0$ and $s=H$ are

$$
j_{\omega}(0, t)=0 \quad \text { and } \quad j_{\omega}(H, t)=0 .
$$


For the plankton flux density, we use the simple approximation

$$
j_{\omega}(s, t)=-D \partial_{s} \omega(s, t)+v \omega(s, t) .
$$

It is composed of an undirected diffusive motion $-D \partial_{s} \omega$ that is driven by the plankton density gradient, and a directed motion $v \omega$. The diffusion can be just Brownian molecular diffusion if the water is macroscopically at rest, or it can mimic turbulent mixing of water. For planktonic cells the second effect is considered dominant, and $D$ is called the turbulent diffusion coefficient (Okubo, 1980). The approximation (3) is valid for relatively low plankton densities, since effects of collective transport or the interaction of hydrodynamic flow fields when several cells are sinking or rising together in the same area, are neglected. Many phytoplankton species lack flagella or cilia, so they cannot swim actively and their motion is passive. This is the case to be considered here. Hence, the directed motion $v \omega$ is due to a specific plankton density different from water. In the absence of diffusion, the drift velocity $v$ can be approximated by Stokes' law (Landau and Lifshitz, 1966; Reynolds, 1984; Denny, 1993)

$$
v=\phi \frac{d^{2}}{18} \frac{\left(\rho_{p}-\rho_{u^{\prime}}\right) g}{\eta}
$$

where $g$ is the earth's gravitational acceleration, $\rho_{p}$ is the specific density of the phytoplankton species concerned, $\rho_{w}$ is the density of water, $\eta$ is the viscosity of water, and $d$ is the diameter of a plankton cell. The parameter $\phi$ is a numerical form factor which takes the particular shape of the object into account. For a spherical cell, we have $\phi=1$. For a species heavier than water, the velocity $v$ is positive, and the motion is downwards. If diffusion is nonvanishing, the laminar flow approximation of equation (4) loses its validity, but dimensional analysis for the mean velocity due to the gravitational force $\alpha\left(\rho_{p}-\rho_{u}\right) g$ still yields a parameter dependence as in (4), but with a different numerical factor $\phi$.

The density $\rho_{p}$, of phytoplankton cells is a species-specific parameter. Species with gas vesicles, like some cyanobacteria, and species with a high oil content, like the green algae Botryococcus, have a lower density than water. They will float upwards $(v<0)$ and will be called buoyant. Because most cell components have a slightly higher density than water, most species that lack gas vesicles have a tendency to sink downwards slowly $(v>0)$. According to the area factor $d^{2}$ in (4), larger cells will move faster than smaller ones. The smallest species, like Chlorella, Synechococcus, and Prochlorococcus, have almost no vertical velocity $(v \approx 0)$.

The source term for the reproduction and death rate in (1) is approximated as

$$
\mathcal{S}(\omega(s, t))=g(I(s, t)) \omega(s, t),
$$

where $g(I(s, t))$ is the specific growth rate of phytoplankton as a function of light intensity $I(s, t)$. This form implies that all nutrients are sufficiently available so 
that the light intensity limits the growth rate. The simplest approximation for such behaviour is

$$
g(I(s, t))=a I(s, t)-\ell,
$$

with a reproduction rate $a I$ proportional to the local light intensity and a constant death rate $\ell$. (Here $\ell$ stands for loss, in agreement with the earlier literature.) This is a good approximation at low light intensities. However, owing to physiological limits of the maximal reproduction rates of organisms, the reproduction rate generally saturates for high light intensities. Such behaviour can be modelled by a function of the form (Monod, 1950)

$$
g(I(s, t))=\frac{a I}{1+c I}-\ell
$$

or alternatively by (Webb et al., 1974; Platt et al., 1980)

$$
g(I(s, t))=a \frac{1-e^{-c /}}{c}-\ell .
$$

For $c l \ll 1$, these functions reduce to the approximation of equation (6). We here work with another general expression

$$
g(I(s, t))=a I^{\alpha}(s, t)-\ell,
$$

that with an appropriate choice for the exponent $0<\alpha \leq 1$ can give a good approximation to (7) or (8). Equation (9) is used because it allows for explicit analytical solutions in terms of Bessel functions. The structure of these solutions turns out to be completely independent of the value of the exponent $\alpha$ as long as $\alpha>0$ and, hence, as long as $g(I)$ is an increasing function of $I$. Besides the numerical evidence, this strongly suggests that our findings do not rely on the particular form of (7), (8), or (9).

As the simplest possible approximation and since typical life and reproduction times are of the order of a day or longer, we assume the light intensity at the surface to take the constant value

$$
I(0, t)=I_{\text {in }} .
$$

Light intensity is decreasing with depth $s$ due to light absorption

$$
\frac{\partial}{\partial s} I(s, t)=-\left(K_{b s}+k \omega(s, t)\right) I(s, t),
$$

where $k$ is the specific light absorption coefficient of the phytoplankton and $K_{h g}$ is the total background absorption due to nonphytoplankton components. The explicit solution of equations (10) and (11) for a given integrated phytoplankton density $\int_{0}^{s} \omega\left(s^{\prime}, t\right) d s^{\prime}$ is

$$
I(s, t)=I_{i n} e^{-K_{l g s} s} e^{-k \int_{0}^{\prime} \omega\left(s^{\prime}, t\right) d s^{\prime}} .
$$


Equations (1), (2), (3), (5), (9), (10) and (11) determine the mathematical problem, together with initial conditions and with the constraint that densities and intensities have to be nonnegative, so

$$
\omega(s, t) \geq 0 \quad \text { and } \quad I(s, t) \geq 0 \quad \text { for all } 0 \leq s \leq H \text { and } t \geq 0 .
$$

\section{Dimensional Analysis and Similarity LaWS}

As it stands now, the model has nine parameters with physical dimensions: the water-column depth $H$, the diffusion constant $D$, the sinking/buoyancy velocity $v$, the parameters $a$ and $\alpha$ in the reproduction rate and the death rate $\ell$, the light intensity $I_{i n}$ at the surface, and the absorption rates $K_{b g}$ and $k$. However, physical phenomena are invariant under the choice of physical units like centimetres or metres. We use this invariance to reduce the number of parameters by means of dimensional analysis. A most convenient choice is to measure length and time scales, plankton density and light intensity as

$$
\begin{gathered}
x=\alpha K_{l g g} s, \quad \tau=D \alpha^{2} K_{b g}^{2} t, \\
\rho(x, \tau)=\frac{k}{K_{b g}} \omega(s, t), \quad j(x, \tau)=\frac{I^{\alpha}(s, t)}{I_{\text {in }}^{\alpha}} .
\end{gathered}
$$

In terms of these rescaled dimensionless variables, the problem depends only on the following four dimensionless parameters

$$
A=\frac{a I_{i n}^{\alpha}}{D \alpha^{2} K_{b g}^{2}}, \quad B=\frac{\ell}{a I_{i n}^{\alpha}}, \quad C=\frac{v}{D \alpha K_{b g}} \quad \text { and } \quad L=\alpha K_{b g} H .
$$

$A$ can be understood as the ratio between the growth rate at the surface and the scales of absorption and diffusion, $B$ is the ratio between death and growth at the surface, $C<0$ measures the buoyancy and $C>0$ the sinking, and $L$ is the dimensionless depth of the water column.

By definition, $A, B$ and $L$ are positive quantities, while the sign of $C$ is not fixed. Additionally, the problem has no nontrivial solution for $B>1$ when the death rate is larger than the growth rate even at the surface $x=0$, as will be proven formally in equation (28). The parameter regime to be explored is, therefore,

$$
0<A<\infty, \quad 0<B<1, \quad-\infty<C<\infty, \quad 0<L<\infty .
$$

In terms of these variables and parameters, the equation for the phytoplankton density defined by (1), (3), (5) and (9), reads

$$
\partial_{\tau} \rho=\partial_{x}^{2} \rho-C \partial_{x} \rho+A(j-B) \rho,
$$


with the boundary conditions (2), (3)

$$
\left[\partial_{x} \rho-C \rho\right]_{x=0}=0, \quad\left[\partial_{x} \rho-C \rho\right]_{x=L}=0 .
$$

The equation for the light intensity (11) becomes

$$
\partial_{x} \ln j=-1-\rho,
$$

with the boundary condition (10)

$$
j(0, \tau)=1 .
$$

The constraint (13) on the nonnegativity of the plankton density now reads

$$
\rho(x, \tau) \geq 0 \quad \text { for all } 0 \leq x \leq L \quad \text { and } \quad \tau \geq 0 .
$$

From equations (20) to (22), it follows immediately, that $j$ is positive and monotonically decreasing towards 0 as $x \rightarrow \infty$, so the constraint (13) on $j$ is automatically obeyed.

The virtue of this dimensional analysis is threefold: (i) it simplifies the equations; (ii) it reveals the similarity laws of different systems: if two systems are characterized by the same four dimensionless variables $A, B, C$, and $L$, they exhibit the same behaviour; (iii) since parameter space is four-dimensional, it can be fully explored and we will pay special attention to the three-dimensional $(A, B, C)$-parameter subspace defined by the limit of infinite water-column depth $L$.

\section{Stationary Solutions and tile Phase Transition}

From here on, we will investigate whether a given set of parameters $(A, B, C, L)$ allows for stationary phytoplankton blooms, i.e., whether there are stationary solutions $\partial_{\tau} \rho=0$ with nonvanishing phytoplankton density. In particular, we will study the critical conditions, where phytoplankton blooms start to exist. Hence we study the stationary solutions of the problem defined by (18)-(22). To simplify the notation, we drop the variable $\tau$ from $\rho$, so we now write $\rho=\rho(x)$. The partial derivatives $\partial_{x}$ then become ordinary derivatives $\mathrm{d}_{x}$. Equations (18)-(21) now constitute a system of one second-order and one first-order nonlinear ordinary differential equation with three boundary conditions. Integration of (20) with (21) leads to a reformulation as one second-order integro-differential equation

$$
\begin{aligned}
\mathrm{d}_{x}^{2} \rho-C \mathrm{~d}_{x} \rho+A\left(e^{-x-\int_{0}^{x} d y \rho(y)}-B\right) \rho & =0, \\
{\left[\mathrm{~d}_{x} \rho-C \rho\right]_{x=0, L}=0, \quad \rho(x) } & \geq 0 \quad \text { for all } 0 \leq x \leq L .
\end{aligned}
$$


A transformation to the variable $R(x)=\int_{0}^{x} d y \rho(y), \rho=d_{x} R$ would lead to a third-order ordinary differential equation with additional boundary value $R(0)=0$, but will not be considered further.

Equation (23) together with (24) for the boundary conditions at $x=0$ and $x=L$ and with the positivity constraint for $\rho(x)$ define a boundary value problem for the phytoplankton density $\rho(x)$. However, it is much more convenient, in particular for the numerics, to consider the problem rather as an initial value problem, where the two initial conditions at $x=0$

$$
\rho(0)=\rho_{0}, \quad\left[\mathrm{~d}_{x} \rho-C \rho\right]_{x=0}=0
$$

fix $L=L\left[\rho_{0}\right]$ as a function of $\rho_{0}, A, B, C$. From here on, we will consider $A, B$ and $C$ fixed, and only write the dependency of $L$ on $\rho_{0}$ explicitly.

If $L\left[\rho_{0}\right]$ exists, then it is unique, as can be seen from the following argument: integrate (23) over $0<x<X$ and use the boundary condition (24) at $x=0$. The result is

$$
\begin{aligned}
{\left[\mathrm{d}_{x} \rho-C \rho\right]_{x=x}=} & -A \int_{0}^{x} d x\left[e^{-x} \mathcal{S}_{\rho}(x)-B\right] \rho(x), \\
& \mathcal{S}_{\rho}(x)=e^{-\int_{0}^{x} d y \rho(y)}
\end{aligned}
$$

where $\rho(x)$ depends parametrically on $\rho_{0}, A, B, C$. Here $\rho(x)$ is positive on the interval according to (24), while $e^{-x} \mathcal{S}_{\rho}(x)$ is monotonically decreasing for growing $x$. A necessary condition for an $X=L\left[\rho_{0}\right]$ to exist is that the function $\left[e^{-x} \mathcal{S}_{\rho}(x)-\right.$ $B]$ changes sign between 0 and $L\left[\rho_{0}\right]$. Hence

$$
0 \leq e^{-L\left[\rho_{0}\right]} \mathcal{S}_{\rho}\left(L\left[\rho_{0}\right]\right) \leq B \leq \mathcal{S}_{\rho}(0)=1
$$

An immediate consequence is that if an $L\left[\rho_{0}\right]$ exists, then the expression $\left[\mathrm{d}_{x} \rho-\right.$ $C \rho]_{x=X}$ is negative for $X<L\left[\rho_{0}\right]$ and positive for $X>L\left[\rho_{0}\right]$. Hence a second solution $L\left[\rho_{0}\right]$ cannot exist and $L\left[\rho_{0}\right]$ is unique.

We are now interested in the phase transition ${ }^{*}$ from bloom to no bloom, in particular in the maximal water depth $L^{*}$, where for given $A, B, C$ phytoplankton can still exist. It is intuitively clear, that a higher plankton density leads to more light absorption, so that the deeper water layers are less favourable for the phytoplankton. One therefore expects that the maximal water depth can be realized for infinitesimal plankton density. This is indeed the case, as we will prove now. More precisely, we will show for the solutions of problem (23)-(25):

\footnotetext{
${ }^{\dagger}$ This phase transition is of second order, hence continuous. Equivalently. in p.d.e. terms, it also can be classified as a supercritical bifurcation. We prefer the notion of a phase transition since we analyse a mean field description of an extended system. Inclusion of fluctuations at a second order phase transition generically leads to slow, but nonhysteretic, relaxation which also is to be expected in the present system.
} 
(1) For a large phytoplankton density $\rho_{0} \gg 1$ at the surface, the water-column depth $L\left[\rho_{0}\right]$ is always finite and unique. In fact, in the limit of $1 / \rho_{0} \rightarrow 0$, it is given explicitly by the positive solution $\mathcal{L}_{1}=\mathcal{L}_{1}(B)$ of the equation

$$
B \mathcal{L}_{1}+e^{-\mathcal{L}_{1}}=1, \quad \text { where } L\left[\rho_{0}\right]=\frac{\mathcal{L}_{1}(B)}{\rho_{0}}+O\left(\frac{1}{\rho_{0}^{2}}\right) .
$$

(2) $L\left[\rho_{0}\right]$ is a monotonically decreasing function of $\rho_{0}$ :

$$
L\left[\rho_{0,1}\right]>L\left[\rho_{0,2}\right], \quad \text { if } \rho_{0,1}<\rho_{0,2} .
$$

Therefore the smaller the phytoplankton density $\rho_{0}$ at the surface, the larger the water-column depth $L\left[\rho_{0}\right]$. As a consequence, for given $A, B$ and $C$, there are two possibilities: either the water-column depth $L\left[\rho_{0}\right]$ becomes infinite already for some nonvanishing value of $\rho_{0}$ or it stays finite up to $\rho_{0} \rightarrow 0$. We then define

$$
L^{*}:=L[0]:=\lim _{\rho_{0} \rightarrow 0} L\left[\rho_{0}\right]
$$

which is unique and given by

$$
L^{*}=\sup _{\rho_{0}>0} L\left[\rho_{0}\right]
$$

In the remainder of the section, these statements are proven.

(1) The result (29) is derived as follows: analyse (23), (24) in the limit of $\rho_{0} \rightarrow$ $\infty$. Rescale $\rho(x)=\rho_{0} r(x)$ and use the new initial condition $r(0)=1$ and $\left.d_{x} r\right|_{0}=C$ instead of (25). The analysis of the exponent in (23) reveals that $1 / \rho_{0}$ introduces a new small length scale into the problem, while on the other hand, $r(x)$ $=1+C x+\cdots$ changes only on the larger scale $1 / C$. Inserting these expressions into (26), $L\left[\rho_{0}\right]$ solves $\left[\mathrm{d}_{x} r-\left.C r\right|_{. x=L\left[\rho_{0}\right]}=0\right.$ and, therefore,

$$
\begin{aligned}
0 & =\int_{0}^{L\left[\rho_{0}\right]} d x\left[e^{-x-\rho_{0} \int_{0}^{X} d y r(y)}-B\right] r(x) \\
\Rightarrow 0 & =\int_{0}^{\mathcal{L}} d X\left[e^{-\frac{X}{\rho_{0}}-\int_{0}^{X} d Y r\left(\frac{y}{\rho_{0}}\right)}-B\right] r\left(\frac{X}{\rho_{0}}\right), \quad r\left(\frac{X}{\rho_{0}}\right)=1+O\left(\frac{\mathcal{L}}{\rho_{0}}\right) \\
& =\int_{0}^{\mathcal{L}} d X\left[e^{-X}-B\right]+O\left(\frac{\mathcal{L}}{\rho_{0}}\right), \quad \text { where } \mathcal{L}=\rho_{0} L\left[\rho_{0}\right] .
\end{aligned}
$$

The evaluation of the last line for $1 / \rho_{0} \rightarrow 0$ and for $\mathcal{L}=\mathcal{L}_{1}(B)+O\left(1 / \rho_{0}\right)$, $\mathcal{L}_{1}(B)=O\left(\rho_{0}^{0}\right)$ immediately yields $(29)$.

(2) The proof of the statements (30) and (32) proceeds along the following steps: first eliminate the first derivative from the equation of motion (23) by the transformation

$$
\rho(x)=\rho_{0} e^{c x / 2} \psi(x),
$$




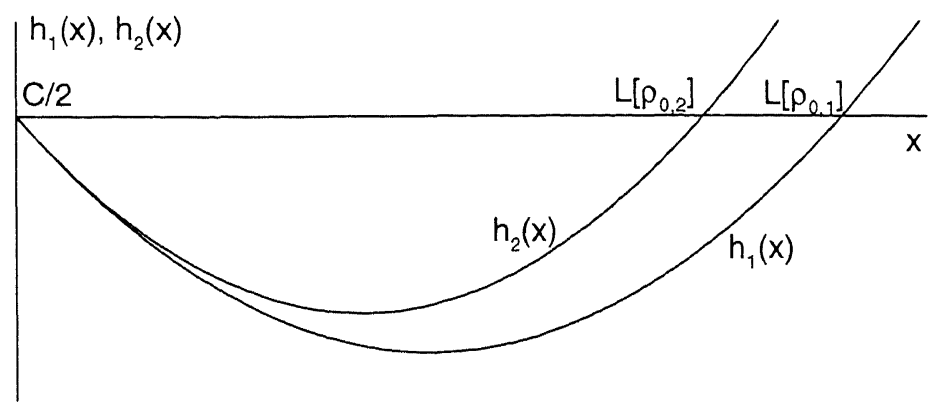

Figure 1. Qualitative behaviour of $h_{1}(x)$ and $h_{2}(x)$ with $\rho_{0,1}<\rho_{0.2}$, together with the resulting $L\left[\rho_{0,1}\right]>L\left[\rho_{0,2}\right]$.

which leads to the transformed equation

$$
\mathrm{d}_{x}^{2} \psi+A\left(e^{-x} \mathcal{S}_{\psi}(x)^{\rho_{0}}-\frac{\nu^{2}}{4 A}\right) \psi=0, \quad v=\sqrt{4 A B+C^{2}},
$$

with the function

$$
\mathcal{S}_{\psi}(x)=\mathcal{S}_{\rho}(x)^{1 / \rho_{0}}=\exp \left[-\int_{0}^{x} d y e^{C y / 2} \psi(y)\right],
$$

and the initial conditions, constraint and definition of $L\left[\rho_{0}\right]$

$$
\psi(0)=1, \quad e^{c x / 2}\left[\mathrm{~d}_{x} \psi-\frac{C}{2} \psi\right]_{0, L\left[\rho_{0}\right]}=0, \quad \psi(x) \geq 0 \quad \text { for } 0 \leq x \leq L\left[\rho_{0}\right] .
$$

The $\psi$-form of the equations will also be the starting point in Section 6 .

For the proof of (30) and (32) it is convenient to perform still another transformation to

$$
\psi(x)=e^{\int_{0}^{x} h(y) d y} \quad \Longrightarrow \quad h(x)=\frac{\mathrm{d}_{x} \psi(x)}{\psi(x)} .
$$

The transformed equation reads

$$
\mathrm{d}_{x} h+h^{2}+A e^{-x} \mathcal{S}(h, x)^{\rho_{0}}-\frac{v^{2}}{4}=0,
$$

with $v$ from (35) and

$$
\mathcal{S}(h, x)=\exp \left[-\int_{0}^{x} d y \exp \left[C y / 2+\int_{0}^{y} d z h(z)\right]\right] .
$$

The initial conditions, constraint and definition of $L\left[\rho_{0}\right]$ now take the form

$$
h(0)=\frac{C}{2}=h\left(L\left[\rho_{0}\right]\right), \quad|h(x)|<\infty \quad \text { for } 0 \leq x \leq L\left[\rho_{0}\right] .
$$


For small $x$, the equation for $h$ can be expanded as

$$
h(x)=\frac{C}{2}-A(1-B) x+A\left(1+C(1-B)+\rho_{0}\right) \frac{x^{2}}{2}+O\left(x^{3}\right) .
$$

So $h(x)$ initially for small positive $x$ decreases below $C / 2$ and eventually has to reach $C / 2$ again from below at $L\left[\rho_{0}\right]$ to obey the condition $h\left(L\left[\rho_{0}\right]\right)=C / 2$.

Compare now two solutions $h_{1}(x)$ and $h_{2}(x)$ of the problem (39)-(41) where $\rho_{0}$ is replaced by $\rho_{0.1}$ and $\rho_{0.2}$, respectively. Assume that $\rho_{0,1}$ is smaller than $\rho_{0.2}$ :

$$
\rho_{0,1}<\rho_{0,2}
$$

The functions $h_{1}(x)$ and $h_{2}(x)$ are shown schematically in Fig. 1. For small $x, h_{2}$ lies above $h_{1}$, since (42) implies that

$$
h_{2}(x)-h_{1}(x)=A\left(\rho_{0,2}-\rho_{0,1}\right) \frac{x^{2}}{2}+O\left(x^{3}\right)>0 \quad \text { for } 0<x \ll 1 .
$$

We will now prove that $h_{2}-h_{1}$ stays positive. The equation for $h_{2}-h_{1}$ is

$$
\mathrm{d}_{x}\left(h_{2}-h_{1}\right)=h_{1}^{2}-h_{2}^{2}+A e^{-x}\left[\mathcal{S}\left(h_{1}, x\right)^{\rho_{0.1}}-\mathcal{S}\left(h_{2}, x\right)^{\rho_{0.2}}\right] .
$$

Now suppose that after an interval $0 \leq x \leq X$ with $h_{2}>h_{1}$, there is a point $x=X$ where $h_{1}=h_{2}$. The expression $\mathcal{S}\left(h_{1}, x\right)^{\rho_{0.1}}-\mathcal{S}\left(h_{2}, x\right)^{\rho_{0.2}}$ at this point $X$ is positive. This is true because $\mathcal{S}\left(h_{2}, x\right)<\mathcal{S}\left(h_{1}, x\right) \leq 1$ according to (40) with $h_{2} \geq h_{1}$ and because $\mathcal{S}^{\rho_{0.2}}<\mathcal{S}^{\rho_{0.1}}$ for all $0<\mathcal{S}<1$ with inequality (43). Therefore for the expression in (45)

$$
\begin{aligned}
& \mathcal{S}\left(h_{1}, x\right)^{\rho_{0.1}}-\mathcal{S}\left(h_{2}, x\right)^{\rho_{0.2}} \\
& \quad=\left[\mathcal{S}\left(h_{1}, x\right)^{\rho_{0.1}}-\mathcal{S}\left(h_{2}, x\right)^{\rho_{0.1}}\right]+\left[\mathcal{S}\left(h_{2}, x\right)^{\rho_{0.1}}-\mathcal{S}\left(h_{2}, x\right)^{\rho_{0.2}}\right] \geq 0 .
\end{aligned}
$$

It follows from (45) that at the point $X$ where we suppose that $h_{2}=h_{1}$, we have $\mathrm{d}_{x} h_{2}>\mathrm{d}_{x} h_{1}$. But this implies that at the crossing point, $h_{2}$ approaches $h_{1}$ from below, which is in contradiction with $h_{2}$ initially being above $h_{1}$. Therefore a crossing point $X$ cannot exist, and

$$
h_{2}>h_{1} \quad \text { for all } x>0 .
$$

Therefore if $h_{2}$ reaches the value $C / 2$ for some $x=L\left[\rho_{0.2}\right]<\infty$, then this value of $x$ will be smaller than the $x=L\left[\rho_{0,1}\right]$ of $h_{1}$. Hence $L\left[\rho_{0,2}\right]<L\left[\rho_{0,1}\right]$ for all

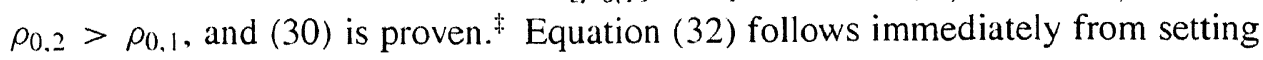
$\rho_{0.1}=0$ and taking the continuity of $L\left[\rho_{0}\right]$ into account.

\footnotetext{
*We thank Lothar Schäfer for helpful discussions in shaping this proof.
} 
We remark that for $1-B \ll 1$, equation (42) immediately yields the explicit approximation

$$
L\left[\rho_{0}\right]=\frac{2(1-B)}{1+\rho_{0}}+O(1-B)^{2}
$$

for small water depth $L$. Of course, this result confirms (30) and (32).

We note finally that our proof of (30) and (32) was performed for a growth rate $g(I)$ as in equations (6) or (9). However, the generalization of (30) and (32) to any function $g(I)$ that is monotonically increasing in $I$ like (7) or (8) is straightforward.

\section{Numerical evaluation of the Phase Transition}

Because of the rigorous bound (32), it is not necessary to study the full nonlinear problem (23), (24) for determining the phase diagram. Rather the maximal watercolumn depth $L^{*}=L[0]$ still allowing for a stationary phytoplankton bloom can be derived from the linear problem defined by

$$
\begin{aligned}
\mathrm{d}_{x}^{2} \rho-C \mathrm{~d}_{x} \rho+A\left(e^{-x}-B\right) \rho & =0, \\
{\left[\mathrm{~d}_{x} \rho-C \rho\right]_{x=0, L^{*}}=0, \quad \rho(x) } & \geq 0 \quad \text { for all } 0 \leq x \leq L^{*},
\end{aligned}
$$

if $L^{*}$ is finite. As equation (49) is linear and the boundary conditions and constraint (50) are homogeneous, the amplitude of $\rho$ is no longer fixed, and the single initial condition $\left[\mathrm{d}_{x} \rho-C \rho\right]=0$ at $x=0$ is sufficient to fix a solution that is unique up to the arbitrary amplitude of $\rho$. This amplitude can be fixed, e.g., by

$$
\rho(0)=1
$$

The two conditions (50) and (51) at $x=0$ together with the second-order equation (49) define an initial value problem that can be integrated numerically towards growing $x$. As also proven, a parameter $L^{*}$ obeying the conditions (50) does not need to exist for fixed $A, B$ and $C$, but if it exists, it is unique.

The data for $L^{*}$ presented in Fig. 2 have been derived by simple numerical integration of this initial value problem for the linear second-order ordinary differential equation (49). Figure 3 could have been derived by extrapolation of the $L^{*} \rightarrow \infty$ lines from Fig. 2, but again we found a much simpler numerical technique for Fig. 3 that will be explained in Section 6.2. We will now discuss these figures in more detail.

A plot of the maximal water depth $L^{*}$ as a function of $A, B$ and $C$ would contain the complete information of the phase transition. But as $\left(A, B, C, L^{*}\right)$ is a fourdimensional space, only projections can be visualized in a three-dimensional plot. In Fig. 2, we have chosen to fix $B$ at the values $0.01,0.2,0.5$ and 0.9 and to plot $L^{*}$ as a function of $A$ and $C$. The choice of fixed $B$ was made, because $B=\ell / a I_{i n}^{\alpha}$ 

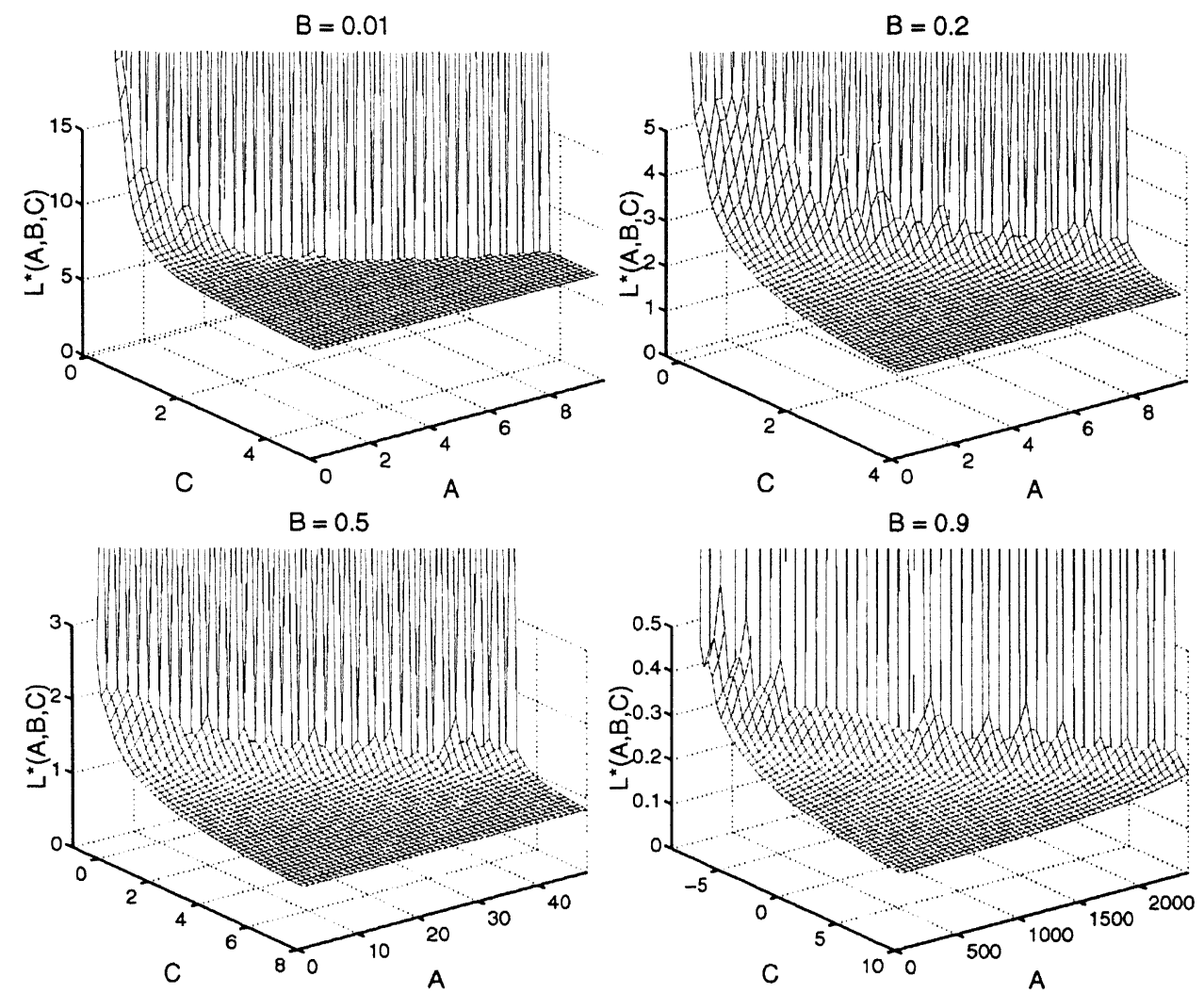

Figure 2. The maximal water-column depth $L^{*}$ as a function of the parameters $A$ and $C$ for fixed values of $B=0.01,0.2,0.5$ and 0.9 . (The slight roughness of the surfaces is a numerical artefact.) For each value of $B$, the maximal water depth $L^{*}$ diverges at a certain curve in the $(A, C)$-plane. These $L^{*} \rightarrow \infty$-curves as a function of $B$ are drawn in Fig. 3.

has a simple interpretation as the ratio of death rate and reproduction rate for a given light intensity $I_{i n}$ at the surface. It will allow for an easy interpretation of the figures, when we return from dimensionless to laboratory parameters in Section 7. For small $A$ and sufficiently large $C$, the maximal water depth $L^{*}$ approaches a constant. This constant value of $L^{*}$ decreases with increasing death rate $B$. Furthermore, for fixed $B$, the maximal water depth $L^{*}$ increases with increasing $A$ and decreasing $C$, and reaches infinity at a critical line in the $(A, C)$-plane.

Figure 3 summarizes the position of the $L^{*} \rightarrow \infty$-lines from Fig. 2 as a function of $B$ in a single plot: it shows the surface where $L^{*} \rightarrow \infty$ in the $(A, B, C)$ parameter space. If a system is characterized by a point $(A, B, C)$ below this surface, a maximal water depth $L^{*}$ does not exist and phytoplankton blooms can develop for any water-column depth $L$. 


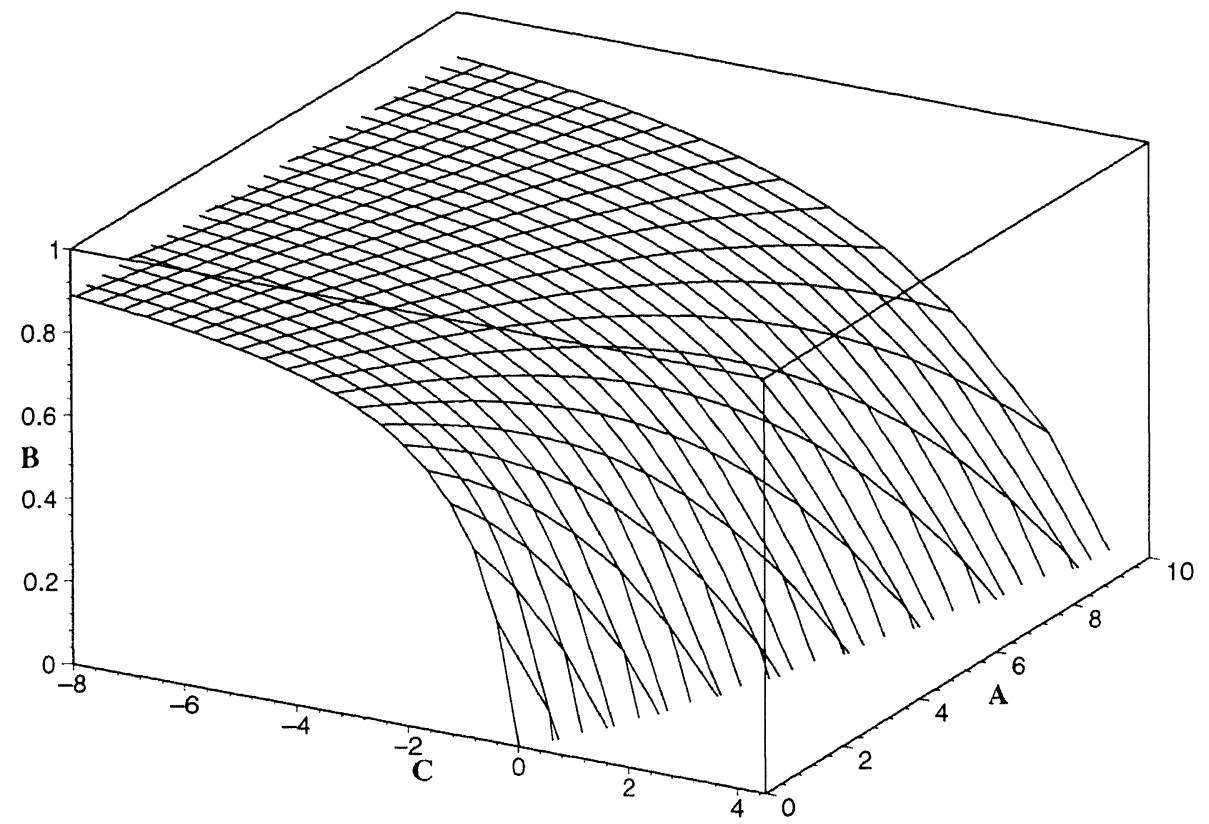

Figure 3. The surface where $L^{*}(A, B, C) \rightarrow \infty$ in $(A, B, C)$-parameter space. The curves on the surface mark either constant $A$ or constant $\nu=\sqrt{4 A B+C^{2}}$. For any combination $(A, B, C)$ below this surface, phytoplankton blooms can exist for any water depth $L$. Conversely, for any combination $(A, B, C)$ above this surface, phytoplankton blooms can exist only if water depth is less than a maximal water depth $L^{*}$.

\section{Analytical Results on the Phase Transition}

The phase transition problem (49), (50) can be studied not only numerically, but also analytically. This will allow the derivation of explicit asymptotes for the surfaces shown in Figs 2 and 3.

6.1. The general criterion. For the analytical study, it is convenient to transform equations (49), (50) first to $\psi(x)=e^{-C x / 2} \rho(x)$ as in (34)-(36). Then the variable $x$ is transformed further as

$$
z^{2}=4 A e^{-x}, \quad \psi(x)=\varphi(z) .
$$

This brings equation (49) into the form of a Bessel equation

$$
z^{2} \frac{\mathrm{d}^{2}}{\mathrm{~d} z^{2}} \varphi(z)+z \frac{\mathrm{d}}{\mathrm{d} z} \varphi(z)+\left(z^{2}-v^{2}\right) \varphi(z)=0, \quad v=\sqrt{4 A B+C^{2}} .
$$

The boundary conditions and constraint (50) now read

$$
z^{C}\left[z \frac{\mathrm{d}}{\mathrm{d} z} \varphi(z)+C \varphi(z)\right]_{z=z_{0,0, z}}=0,
$$




$$
\begin{aligned}
& \text { at } \quad z_{0}=\sqrt{4 A}, \quad z_{1}=z_{0} e^{-L^{*} / 2}, \\
& \text { and } \quad \varphi(z) \geq 0 \quad \text { for } z_{1} \leq z \leq z_{0} .
\end{aligned}
$$

The solution $L^{*}$ is unique if it exists, since we analyse a transformed but equivalent version of the problem studied in Section 4. The general solution of (53) is given by the superposition of two Bessel functions

$$
\varphi(z)=c_{1} J_{1^{\prime}}(z)+c_{2} Y_{v}(z)
$$

where $J_{v^{\prime}}(z)$ and $Y_{v}(z)$ are the Bessel functions of order $v$ of first and second kind, respectively, and $c_{1}$ and $c_{2}$ are constants of integration. For the general properties of the Bessel functions, their notation, and for a number of explicit expansions and identities that will be used later, we refer to Abramowitz and Stegun (1964). In particular, the function $J_{11}(z)$ has the expansion

$$
J_{1}(z)=\left(\frac{z}{2}\right)^{\prime} \sum_{n=0}^{\infty} \frac{\left(-z^{2} / 4\right)^{n}}{n ! \Gamma(v+n+1)}
$$

where $\Gamma(x)$ is the $\Gamma$-function. The function $Y_{v}(z)$ is related to $J_{1}$ and $J_{-v^{\prime}}$ as

$$
Y_{11}(z)=\frac{\cos v \pi J_{11}(z)-J_{-1}(z)}{\sin v \pi}
$$

Therefore for small argument $z$ and for $\nu>0, J_{v}$, vanishes like $z^{v}$, while $Y_{v}$ and $J_{-\nu}$ diverge like $z^{-v}$.

The ratio $c_{2} / c_{1}$ of the arbitrary constants $c_{1}$ and $c_{2}$ in (57) is fixed by the boundary conditions (54):

$$
-\frac{c_{2}}{c_{1}}=\left.\frac{\left(z \mathrm{~d}_{z}+C\right) J_{1}(z)}{\left(z \mathrm{~d}_{z}+C\right) Y_{1}(z)}\right|_{z_{0}}=\left.\frac{\left(z \mathrm{~d}_{z}+C\right) J_{1}(z)}{\left(z \mathrm{~d}_{z}+C\right) Y_{1}(z)}\right|_{z=1}
$$

Elimination of $c_{2} / c_{1}$ leads to the determinant condition on $\nu, C, z_{0}$ and $z_{1}$

$$
\Delta:=\left|\begin{array}{ll}
\left.\left(z \mathrm{~d}_{z}+C\right) J_{v}(z)\right|_{z_{0}} & \left.\left(z \mathrm{~d}_{z}+C\right) Y_{v}(z)\right|_{z 0} \\
\left.\left(z \mathrm{~d}_{z}+C\right) J_{v}(z)\right|_{z 1} & \left.\left(z \mathrm{~d}_{z}+C\right) Y_{v}(z)\right|_{z 1}
\end{array}\right|=0
$$

Equation (60) or (61) together with the positivity constraint (56) for the function (57) define the phase transition from bloom to no bloom.

The original variables $A, B, C$ and $L^{*}$ can be recovered from $\nu, C, z_{0}$ and $z_{1}$ by

$$
A=\frac{z_{0}^{2}}{4}, \quad B=\frac{\nu^{2}-C^{2}}{z_{0}^{2}}, \quad C=C, \quad L^{*}=2 \ln \frac{z_{0}}{z_{1}}
$$


6.2. The limit $L^{*} \rightarrow \infty$. For systems with an infinite water-column depth, the condition takes a simpler form. This can be seen by inserting the small $z$ asymptotics of the Bessel functions (58) into (60). Evaluation of the expression for $L^{*} \rightarrow \infty$ corresponds to $z_{1} \rightarrow 0$. One finds

$$
-\frac{c_{2}}{c_{1}}=\left.\lim _{z_{1} \rightarrow 0} \frac{\pi(\nu+C)(z / 2)^{\nu}}{(\nu-C)(z / 2)^{-v} \Gamma(\nu+1) \Gamma(v)}\right|_{z=1}=0 \quad \text { for } A B>0
$$

since $v=\sqrt{4 A B+C^{2}}>|C|$ for $A B>0$ and the parameter regimes given in (17). [In particular in view of the limit $z \rightarrow 0$, the factors $e^{C x / 2}$ or $z^{-C}$ have been kept in (37) and (54), and we remark that the full expression (54) also vanishes at $z=0$, if and only if $c_{2}=0$.] Inserting $c_{2}=0$ from (63) in equation (57), $\varphi(z)$ is found to be proportional to $J_{1,}(z)$, and the boundary condition at $z_{0}$ reduces to

$$
\left.\left(z \mathrm{~d}_{z}+C\right) J_{v}(z)\right|_{z_{0}}=\left.0 \quad \Longleftrightarrow \quad \frac{\mathrm{d} \ln J_{v}(z)}{\mathrm{d} \ln z}\right|_{z_{0}}=-C \quad \text { for } L^{*} \rightarrow \infty
$$

with the positivity constraint

$$
J_{v}(z) \geq 0 \quad \text { for } 0 \leq z \leq z_{0} .
$$

Now the criterion (64) and (65) is further evaluated. Let us introduce, in particular, the function

$$
f_{v}(z):=\frac{\mathrm{d} \ln J_{v}(z)}{\mathrm{d} \ln z}=\frac{z J_{v^{\prime}-1}(z)}{J_{v}(z)}-v,
$$

where the last identity results from the general relation $J_{v}^{\prime}(z)=J_{\nu-1}(z)-\nu J_{v}(z) / z$ between Bessel functions. $f_{\mathrm{v}}(z)$ solves the first order nonlinear equation

$$
\mathrm{d}_{z} f_{v}(z)=\frac{v^{2}-f_{v}^{2}(z)-z^{2}}{z}, \quad f_{v}(0)=v
$$

[ $f_{\nu}(z)$ is related to the function $h_{0}(x)$ from Section 4 by $f_{1}(z)=-2 h_{0}(x)$ and the relation (52) between $x$ and $z$.J $z_{0}$ is now determined by the constraint (65) and

$$
f_{v}\left(z_{0}\right)=-C \text {. }
$$

We remark that the $L^{*} \rightarrow \infty$ solutions in Fig. 3 have actually been generated by varying $v$ and $z_{0}$ in (66) and calculating $C$ from (68). The lines in Fig. 3 are lines of constant $A$ and $\nu$. The relation of these parameters to the parameters $(A, B, C)$ is given in (62).

For the further analytical progress, it is easy to realize either from Abramowitz and Stegun (1964) or from a construction of the flow of $(67)$, that $f_{1}(z)$ is a monotonically decreasing function of $z$. Since $|C|<v$ by construction, the limiting values for $z_{0}$ are

$$
-C=\nu \leftrightarrow z_{0}=0, \quad C=0 \leftrightarrow z_{0}=j_{\nu, 1}^{\prime}>\nu, \quad C=\nu \leftrightarrow z_{0}=j_{v-1,1},
$$

with $j_{v, 1}^{\prime}$ and $j_{v, 1}$ the first zero of $J_{v}^{\prime}(z)$ and $J_{v}(z)$ for positive $z$. 
In the limits $z_{0} \ll 1$ and $z_{0} \gg 1$, several asymptotic expansions can be given. For $z_{0}^{2}=4 A \ll 1$, we get either from (67) or from Abramowitz and Stegun (1964)

$$
-C=\nu-\frac{z_{0}^{2}}{2(1+\nu)}-\frac{z_{0}^{4}}{8(1+\nu)^{2}(2+\nu)}-\frac{z_{0}^{6}}{16(1+\nu)^{3}(2+\nu)(3+\nu)}+O\left(z_{0}^{8}\right) .
$$

If also $|C| \ll 1$, we get from expression (70) in lowest order that $B=A-C$. So $B$ also has to be small, if $A$ and $C$ are small, cf. Fig. 3. Further expansion with $B=A-C+O(A+C)^{2}$ yields

$$
\begin{aligned}
B= & A-C-\frac{(3 A-2 C)(2 A-C)}{2}+\frac{143 A^{3}-208 A^{2} C+93 A C^{2}-12 C^{3}}{12} \\
& +O\left(A^{4}\right)+O\left(A^{3} C\right)+\cdots+O\left(C^{4}\right) \quad \text { for } A \ll 1 \quad \text { and } \quad|C| \ll 1 .
\end{aligned}
$$

The expansion of (70) for $A \ll 1$ and an arbitrary negative value of $-C=|C| \gg$ $\sqrt{4 A B}$ is

$$
\begin{aligned}
& B=\frac{|C|}{1+|C|}+\frac{2 A}{(1+|C|)^{3}(2+|C|)}+O\left(A^{2}\right) \\
& \quad \text { for } A \ll 1, \quad C<0 \quad \text { and } \quad A \ll \frac{|C|(1+|C|)}{4} .
\end{aligned}
$$

For $A \ll 1$ and $C \gg \sqrt{4 A B}$, there is no solution with positive $B$.

For $z_{0}^{2}=4 A \gg 1$, also $v \gg 1$ because of the bound $z_{0} \leq j_{\nu-1,1}=O(v)$ from (69). Expressing the Bessel function $J_{11}(z)$ for argument $z_{0}=v-x(v / 2)^{1 / 3}$ by $\operatorname{Airy}$ functions $\operatorname{Ai}(x)$ yields the large $v$ expansion

$$
z_{0}=v-\left(\frac{v}{2}\right)^{1 / 3} \times\left[\frac{C}{v^{2 / 3}}\right]+O\left(\frac{1}{v^{1 / 3}}\right)
$$

where $x\left[C / v^{2 / 3}\right]$ is defined implicitly by

$$
\frac{C}{v^{2 / 3}}=2^{1 / 3} \frac{d \ln \operatorname{Ai}(x)}{d x}
$$

and $w(x)=\mathrm{Ai}(x)$ is the solution of $\mathrm{d}_{x}^{2} w=x w$ with $\lim _{x \rightarrow \infty} w(x)=0$.

There are two limiting values for $x$, namely $x \approx-1.0$ for $|C| \ll v^{2 / 3}$ and $x \approx-2.3$ for $C \gg v^{2 / 3}$. [For $|C| \gg v^{2 / 3}$ and $C<0, z_{0}$ eventually becomes so small that the ansatz (73) loses its validity.] Insertion of (62) into (73) for a fixed value of $x$ results in

$$
B=1-\frac{C^{2}}{4 A}+\frac{x}{A^{1 / 3}}+O\left(\frac{1}{A^{2 / 3}}\right) \quad \text { for } A \gg 1 \quad \text { and } \quad C \geq 0 .
$$


Note that $C$ can become large, while $-2.3 \leq x \leq-1.0$ for all $C \geq 0$.

We finally remark that for $L^{*} \gg 1$, the expansion (63) for $z_{1}^{2}=4 A e^{-L^{*}} \ll 1$ inserted into the general relation (60) yields the expansion about the limit (64) of $L^{*} \rightarrow \infty$

$$
\begin{aligned}
\left.\frac{\left(z \mathrm{~d}_{z}+C\right) J_{v}(z)}{\left(z \mathrm{~d}_{z}+C\right) Y_{v}(z)}\right|_{z_{0}=\sqrt{4 A}}= & \left(A e^{-L^{*}}\right)^{\nu} \frac{\pi(v+C)}{(v-C) \Gamma(v+1) \Gamma(v)} \\
& +O\left(A e^{-L^{*}}\right)^{v+1} .
\end{aligned}
$$

The asymptotic expansions (71), (72) and (75) provide simple approximations to the surface shown in Fig. 3.

\section{Back to the Original Parameters}

Let us return from the dimensionless variables $A, B, C$ and $L$ defined in (16) to the original variables. These are the death rate $\ell$, the parameters $a$ and $\alpha$ in the growth rate (9), the sinking velocity $v$ and the specific light absorption coefficient $k$ of the phytoplankton, and the incident light intensity $I_{i n}$, the background turbidity $K_{b \xi}$, the diffusion constant $D$ and the depth $H$ of the water basin.

7.1. Critical diffusion and water-column depth. We now study the effect of the diffusion constant $D$ on the phase transition. It was absorbed into the dimensionless parameters $A$ and $C$. Hence we now define

$$
A_{0}=A D=\frac{a l_{i n}^{\alpha}}{\alpha^{2} K_{b s}^{2}} \quad \text { and } \quad C_{0}=C D=\frac{v}{\alpha K_{b s}} .
$$

The parameters $A_{0}, C_{0}$ and $D$ all have the physical dimension of a diffusion constant. A variation of the parameter $D$ for fixed light intensity $I_{i n}$ amounts to a variation of $A$ and $C$ with fixed value of $B$ and fixed ratio

$$
\gamma=\frac{C}{A}=\frac{C_{0}}{A_{0}}=\frac{v \alpha K_{b s}}{a I_{i n}^{\alpha}}=\frac{v \alpha K_{b s}}{\ell} B
$$

Possible phase transition scenarios as a function of diffusion constant $D$ and water depth $L$ where all other parameters are fixed, will be presented in Fig. 5. In particular, we will discuss the case $B=0.5$ as an example, i.e., the case when the incident light intensity is related to the growth and death rate like $\ell=0.5 a I_{i n}^{\alpha}$.

Figure 5 can be derived by a simple projection of Fig. 2. To illustrate and explain this procedure, we introduce Fig. 4 as an intermediate step. The solid curves in Fig. 4 represent the data of Fig. 2 for $B=0.5$ as level curves of constant $L^{*}$ in the $(A, C)$ plane; the fat solid curve marks the divergence of the maximal water 


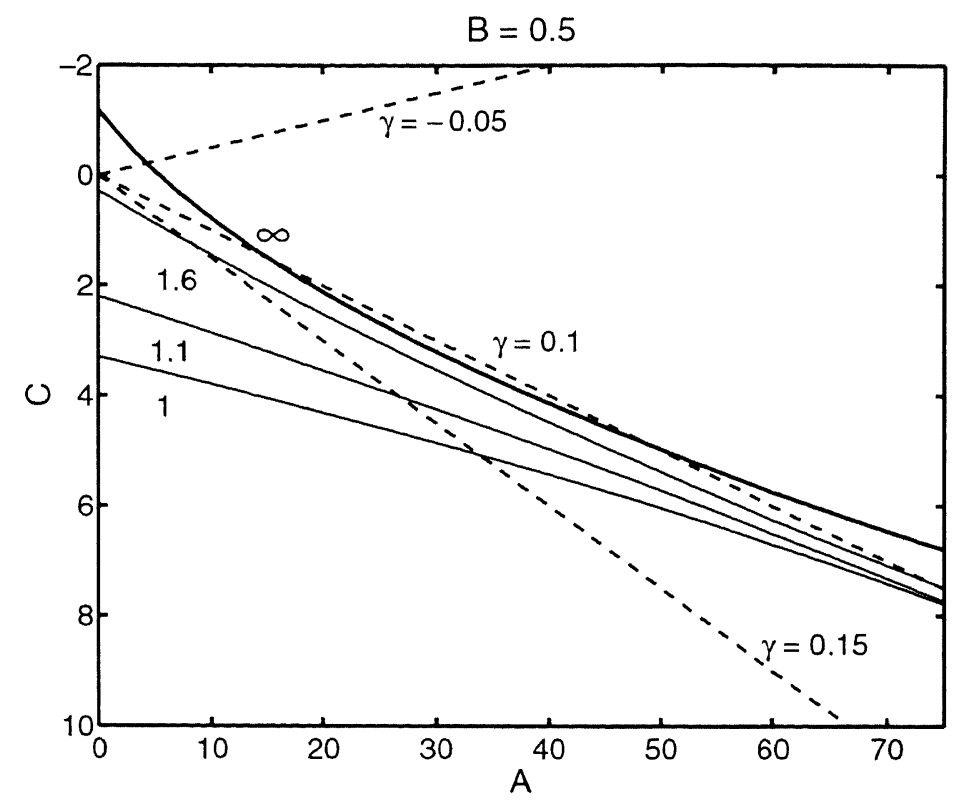

Figure 4. The solid curves in the $(A, C)$-plane are level curves of constant $L^{*}(A, B, C)$ for $B=0.5$. They present the data of Fig. 2(c). The fat solid curve denotes $L^{*} \rightarrow \infty$, the thin solid curves $L^{*}=1.6,1.1$ and 1 . For any combination $(A, C)$ above the fat solid curve, phytoplankton blooms will exist independent of water-column depth. Conversely, for any combination $(A, C)$ below the fat solid curve, phytoplankton blooms can exist only if water-column depth is less than $L^{*}(A, B, C)$. The three straight dashed lines starting at the origin are lines of fixed ratio $\gamma=C / A$ with the values $\gamma=-0.05,0.1$ and 0.15 . Variation along these dashed lines implies that only the diffusion constant $D$ is varied, whereas all other model parameters are kept constant.

depth $L^{*}$, while the thin solid curves denote finite values of $L^{*}$. The straight dashed lines starting from the origin are lines of constant $\gamma$ with the values $\gamma=-0.05$ for a buoyant phytoplankton species and $\gamma=0.1$ and 0.15 for two species with different sinking rates. These three dashed lines represent three different possible behaviours: first, all lines with $\gamma \leq 0$ (buoyant or neutrally buoyant species) intersect with the $L^{*} \rightarrow \infty$-curve precisely once. This intersection point indicates the value of the turbulent diffusion constant at which the maximal water-column depth $L^{*}$ diverges. Hence, for buoyant or neutrally buoyant phytoplankton, there is precisely one critical value of the turbulent diffusion coefficient. Second, the $\gamma=0.1$-line intersects twice with the $L^{*} \rightarrow \infty$-curve. This indicates that there is a critical value of the diffusion constant at which the maximal water-column depth diverges, and another value of $D$, below which the maximal water-column depth again becomes bounded. Third, the $\gamma=0.15$-line does not intersect with the $L^{*} \rightarrow \infty$-curve at all. In this case, there is no critical value of $D$; rather the maximal water-column depth is bounded for all values of $D$. Thus, for sinking phytoplankton, there are either two critical values of the turbulent diffusion constant 

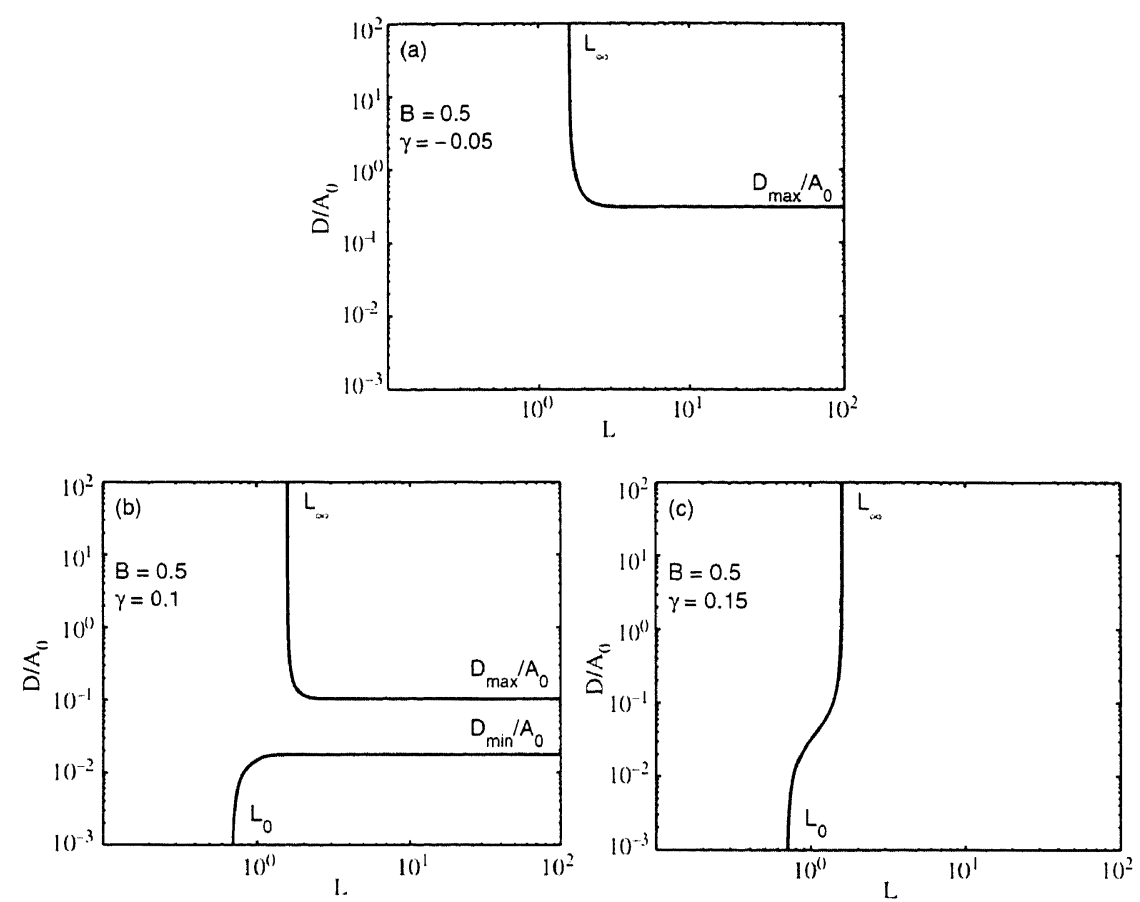

Figure 5. The phase boundary of phytoplankton bloom development, plotted as a function of water-column depth $L=\alpha K_{h g} H$ and scaled diffusion constant $D / A_{0}$. Phytoplankton bloom always exists for sufficiently shallow water columns $L \ll 1$. The phase boundary in the plane of $L$ and $D / A_{0}$ depends on $B$ and $\gamma$. Here it is shown for $B=0.5$ and three values of $\gamma$. The plots are projections of Fig. 2 (with Fig. 4 as an intermediate step) onto different plankton species with $\gamma=-0.05$ in (a), $\gamma=0.1$ in (b) and $\gamma=0.15$ in (c). We indicated the asymptotic limits of critical depth $L_{\infty}$ at $D \rightarrow \infty$, compensation depth $L_{0}$ at $D \rightarrow 0$, and maximal and minimal critical diffusion $D_{\max }$ and $D_{\min }$ at $L^{*} \rightarrow \infty$.

or none at all. To be more precise, between 0.1 and 0.15 , there is a $\gamma$-line tangent to the $L^{*} \rightarrow \infty$-curve, where the two intersection points merge and disappear. We denote this particular value of $\gamma$ as $\gamma_{c}(B)$.

Figure 5 shows the phase boundary of phytoplankton bloom development as a function of the water-column depth $L=\alpha K_{b g} H$ and the scaled diffusion constant $D / A_{0}$. It should be remarked that these plots depend on the two parameters $\gamma$ and $B$ only, and that the same values of $\gamma$ and $B$ as in Fig. 4 have been chosen. The projection procedure from Fig. 4 to Fig. 5 is as follows: the $A$-axis of Fig. 4 is inverted to give the $1 / A=D / A_{0}$-axis of Fig. 5. The values of $L^{*}(A, B, C)$ along a line of constant $\gamma$ and $B$ in Fig. 4 are plotted on the $L$-axis of Fig. 5.

The values of $\gamma$ have been chosen to illustrate the three different possible forms of the $(D, L)$ diagrams. Figure 5(a) with $\gamma=-0.05$ is representative for all nonpositive values of $\gamma$, i.e., for buoyant or neutrally buoyant phytoplankton. As can be seen in Fig. 4 , a line of constant $\gamma$ intersects with each value of $L^{*}$ exactly once. Hence the maximal water depth $L^{*}$ is a monotonically decreasing function of $D$, 
and there is only one critical value of $D$ for $L^{*} \rightarrow \infty$, called the maximal critical diffusion $D_{\max }$. For sinking phytoplankton, i.e., for $\gamma>0$, there are two possibilities. Figure 5(b) shows the data of Fig. 4 projected onto $\gamma=0.1$. Here there are two critical values of $D$ for $L^{*} \rightarrow \infty$. We will call these two values the minimal and the maximal critical diffusion, $D_{\min }$ and $D_{\max }$, respectively. For all values of $D$ between $D_{\min }$ and $D_{\max }$, a bounded maximal water-column depth does not exist, and, hence, phytoplankton blooms can develop in any water-column depth. This figure is representative for all positive $\gamma$ smaller than $\gamma_{c}(B)$. Figure 5(c) shows the data for $\gamma=0.15$, which implies that $\gamma$ is larger than $\gamma_{c}(B)$. In this case, there is no critical value of $D$ for $L^{*} \rightarrow \infty$. That is, there is always a bounded maximal water-column depth $L^{*}$, whatever the value of the diffusion coefficient $D$.

We note that the limit value $L_{\infty}$ for $D \rightarrow \infty$ is the same in all three panels in Fig. 5. This can be understood immediately from Fig. 4, since it corresponds to the value of $L^{*}(A, B, C)$ in the point $(A, C) \rightarrow(0,0)$ that is reached by all $\gamma$ lines. Also the limit value $L_{0}$ for $D \rightarrow 0$ is the same for all positive values of $\gamma$. It corresponds to the saturation value of $L^{*}$ in Fig. 4 for large $A$ and fixed ratio $\gamma=C / A>0$.

In the remainder of this section, we will derive analytical results for the critical values $D_{\max }$ and $D_{\min }$ in the limit $L^{*} \rightarrow \infty$, and for the critical values $L_{0}$ and $L_{\infty}$ in the limits of $D \rightarrow 0$ and $D \rightarrow \infty$. We also present asymptotic expansions about these limits.

7.2. Compensation depth: the limit $D \rightarrow 0$. What happens if turbulent diffusion is negligibly small? In this case, the motion of phytoplankton is governed by buoyancy or sinking only. Hence, in the long run, buoyant phytoplankton will completely float at the surface, so the depth of the water column below it does not play any role, if only at the surface the growth rate is larger than the death rate. Thus, for $\gamma<0$, there is phytoplankton bloom development whenever $B<1$, and hence there is no critical water-column depth $L_{0}$ if diffusion is low [Fig. 5(a)].

In contrast, sinking phytoplankton will sink to the bottom of the water column if diffusion is negligibly small, and hence they will survive only if the reproduction rate at the bottom overcomes the death rate. Thus, for sinking phytoplankton, there always exists a maximal water-column depth at low diffusion, defined earlier as the compensation depth $L_{0}$ [Fig. 5(b) and 5(c)]. In terms of equations (9) and (16), the compensation depth $L_{0}=\alpha K_{b g} H_{0}$ is given by

$$
g\left(I_{\text {in }} e^{-K_{\text {lg }} H_{0}}\right)=0 \quad \Longleftrightarrow \quad L_{0}(B)=-\ln B \quad \text { for } \gamma>0, \quad D=0 .
$$

The calculation presented in this subsection reproduces this result and extends it with a small $D$ expansion:

$$
e^{-L^{*}}=B\left(1-\frac{1}{\gamma} \frac{D}{A_{0}}+O\left(\frac{D}{A_{0}}\right)^{3}\right) \quad\left(\gamma A_{0}=C_{0}\right)
$$




$$
\Leftrightarrow L^{*}=-\ln B+\frac{1}{\gamma} \frac{D}{A_{0}}+\frac{1}{2 \gamma^{2}}\left(\frac{D}{A_{0}}\right)^{2}+O\left(\frac{D}{A_{0}}\right)^{3} \text { for } \gamma>0, \quad D \rightarrow 0 .
$$

In the remainder of the section, we will derive this result from (61) and (62).

$\nu$ and $z_{0}$ are expressed by $A_{0}, B, \gamma=C_{0} / A_{0}>0$ and $D$ as

$$
v=\gamma \frac{A_{0}}{D} \sqrt{1+\frac{D}{A_{0}} \frac{4 B}{\gamma^{2}}}, \quad z_{0}=\sqrt{\frac{4 A_{0}}{D}}
$$

Hence for fixed $A_{0}, B$ and $\gamma>0$ and for $D \rightarrow 0$, we always have $\nu \gg 1$ and $z_{0} \ll v$. Furthermore $z_{1}=z_{0} e^{-L^{*} / 2} \leq z_{0}$. Therefore for the evaluation of the Bessel functions in (61), Debye's asymptotic expansions can be used:

$$
\begin{aligned}
J_{v}(z) & \sim \frac{e^{v x}}{\sqrt{2 \pi v t}}\left(1+\sum_{k=1}^{\infty} \frac{u_{k}(t)}{v}\right), \quad Y_{\nu}(z) \sim \frac{-2 e^{-v x}}{\sqrt{2 \pi v t}}\left(1+\sum_{k=1}^{\infty} \frac{u_{k}(t)}{(-v)^{k}}\right) \\
t & =\left(1-\frac{z^{2}}{v^{2}}\right)^{-1 / 2}, \quad \chi=t-\operatorname{arcosh} \frac{v}{z} \approx \stackrel{\approx}{\approx}-\ln \frac{2 v}{z}+O(1)
\end{aligned}
$$

The $u_{k}(t)$ are polynomials of degree $3 k$ in $t$, that can be found in Abramowitz and Stegun (1964). Insertion of these expansions into the determinant (61) leads to the prefactors $e^{\nu\left(x\left(z_{0}\right)-x\left(z_{1}\right)\right)} \approx\left(z_{1} / z_{0}\right)^{\prime \prime}=e^{-v L^{*} / 2}$ and $e^{-v\left(x\left(z_{0}\right)-x\left(z_{1}\right)\right)} \approx e^{v L^{*} / 2}$. For any finite $L^{*}$ and $\nu \rightarrow \infty$, these factors dominate the expressions in (61). Further evaluation similar to (63), (64) shows that in the limit of vanishing diffusion constant and for $C>0, L^{*}$ as a function of $A, B$ and $C$ is determined by

$$
\left.\left(z \mathrm{~d}_{z}+C\right) Y_{n}(z)\right|_{z_{1}}=0
$$

This equation has a solution for $D \rightarrow 0$ only if $C>0$. Evaluating (83) further with the help of (82) gives a functional relation between the three quantities

$$
\lambda=1-\frac{e^{-L^{*}}}{B}, \quad \delta=\frac{1}{C}=\frac{D}{\gamma A_{0}} \quad \text { and } \quad \epsilon=\frac{A B}{C^{2}}=\frac{D}{A_{0}} \frac{B}{\gamma^{2}}=\delta \frac{B}{\gamma},
$$

since the parameters in (83) can be written as

$$
C=\frac{1}{\delta}, \quad v=\frac{\sqrt{1+\epsilon}}{\delta}, \quad t\left(\frac{z_{1}}{v}\right)=\sqrt{\frac{1+\epsilon}{1+\epsilon \lambda}} .
$$

Evaluating (83) up to order $D^{2}$, the $D$-expansion of $\lambda$ is $\lambda=\delta+O\left(D^{3}\right)$. Insertion of (84) yields our final result (80). 
7.3. Critical depth: the limit $D \rightarrow \infty$. What happens if mixing by turbulent diffusion becomes infinitely fast? In this case, the phytoplankton is homogeneously distributed through the whole water column, and no spatial structures remain. Hence the critical depth $L_{\infty}=\alpha K_{h g} H_{\infty}$ as defined by Sverdrup (1953) is reached if the growth rate integrated over the whole column balances the death rate for constant phytoplankton density

$$
\int_{0}^{H_{\infty}} g\left(I_{i n} e^{-K_{l g s} s}\right) d s=0 \quad \Leftrightarrow \quad B=\frac{1-e^{-L_{\infty}}}{L_{\infty}} \quad \text { for } D \rightarrow \infty .
$$

Here the critical depth $L_{\infty}=L_{\infty}(B)$ is the positive solution of the above equation. In the remainder of this subsection, we will expand in the small parameter $1 / D$ about the asymptotic behaviour for $D \rightarrow \infty$. The result of this calculation can be written as

$$
L^{*}=L_{\infty}(B)+\frac{A_{0}}{D} L_{1}(B, \gamma)+\left(\frac{A_{0}}{D}\right)^{2} L_{2}(B, \gamma)+\cdots,
$$

where $L_{1}$ is given by

$$
\begin{aligned}
L_{1}(B, \gamma)=\frac{L_{\infty}}{6\left(B-1+B L_{\infty}\right)} \times\left[3 \gamma\left(B L_{\infty}-2(1-B)\right)\right. \\
\left.+\left(2 B^{2} L_{\infty}^{2}+3 B(3 B-2) L_{\infty}+6(2 B-1)(B-1)\right)\right] .
\end{aligned}
$$

Let us first discuss the consistency and implications of this result: if the death rate at the surface almost equals the growth rate, i.e., if $B \uparrow 1$, then $L_{\infty}=$ $2(1-B)+O(1-B)^{2}$ is small and the expansion (87) reproduces the earlier result (48). If the death rate at the surface is negligible, i.e., if $B \downarrow 0$, then the water depth diverges like $L_{\infty}=1 / B+O\left(e^{-1 / B} / B\right)$. Furthermore we remark that $L_{\infty}>L_{0}$ from (79) for all $B$ and $C>0$ : that is, the critical depth is always larger than the compensation depth since phytoplankton distributed over the whole water column has better reproduction conditions than phytoplankton at the bottom.

The results (86)-(88) are now derived from the determinant (61). For $D \rightarrow \infty$, the parameters $z, v$ and $C$ are small. Expressed in terms of the small parameter $z_{0}^{2}=4 A=4 A_{0} / D$ and the fixed parameters $\gamma=C_{0} / A_{0}, B$ and $L^{*}$, they read

$$
z_{1}^{2}=e^{-L^{*}} z_{0}^{2}, \quad C=\gamma z_{0}^{2} / 4, \quad \nu^{2}=B z_{0}^{2}+\gamma^{2} z_{0}^{4} / 16 .
$$

Since $z_{1} \leq z_{0} \ll 1$, the determinant (61) can now be evaluated with the asymptotic expansions (58) and (59):

$$
\begin{aligned}
\Delta & =-\frac{e^{\nu L^{*} / 2}}{\nu \pi} p_{v}\left(z_{0}\right) p_{-v}\left(z_{1}\right)+\frac{e^{-v L^{*} / 2}}{v \pi} p_{-v}\left(z_{0}\right) p_{\nu}\left(z_{1}\right) \\
p_{\nu}(z) & =\sum_{n=0}^{\infty} \frac{(2 n+\nu+C)\left(-z^{2} / 4\right)^{n}}{n ! \prod_{k=1}^{n}(k+\nu)} .
\end{aligned}
$$


A short consideration shows that $\Delta(-v)=\Delta(v)$, so $\Delta$ is actually a function of $v^{2}$, and therefore the expansion of $\Delta$ orders in powers of $1 / D$, not $1 / \sqrt{D}$. With the ansatz (87) and

$$
\Delta=\frac{1}{\nu \pi D \sqrt{D}}\left[\Delta_{0}+\frac{\Delta_{1}}{D}+\frac{\Delta_{2}}{D^{2}}+\cdots\right]
$$

the $L_{k}$ can be solved hierarchically from $\Delta_{k}=0$ for all $k$. Since $\Delta_{0}=0$ for

$$
1-e^{-L_{\infty}}-B L_{\infty}=0,
$$

the result (86) for $L_{\infty}$ follows immediately. $\Delta_{1}=0$ determines $L_{1}$, the result (88) was given earlier.

7.4. Critical diffusion: the limit $L^{*} \rightarrow \infty$. The case of diverging water depth $L^{*} \rightarrow \infty$ as a function of $A, B$ and $C$ was already analysed in detail in Section 6.2: in general, the relation between $A, B$ and $C$ for infinite water depth is given by

$$
\frac{z_{0} J_{v-1}\left(z_{0}\right)}{J_{v}\left(z_{0}\right)}=v-C \quad \text { with } \quad v=\sqrt{4 A B+C^{2}} \quad \text { and } \quad z_{0}=\sqrt{4 A} \text {. }
$$

The critical diffusion $D$ as a function of $A_{0}, B$ and $C_{0}$ results from replacing $A$ by $A_{0} / D$ and $C$ by $C_{0} / D=\gamma A_{0} / D$. The identity (93) yields both the maximal critical diffusion $D_{\max }$ and the minimal critical diffusion $D_{\min }$, if they exist. However, the relation is implicit, and explicit predictions for the critical diffusion can only be derived by asymptotic expansions valid in some part of parameter space. Depending on the values of $B$ and $\gamma=C_{0} / A_{0}$, these expansions take different forms. We only consider a few special cases with the following explicit results:

For $z_{0}^{2}=4 A_{0} / D \ll 1$, equation (93) is approximated by equation (70). If, furthermore, $B \ll 1$ and $\gamma$ of order unity, the diffusion constant according to (71) diverges like

$$
\begin{gathered}
\frac{D_{\max }}{A_{0}}=\frac{1-\gamma}{B}-\frac{(3-2 \gamma)(2-\gamma)}{2(1-\gamma)}+B \frac{35-99 \gamma+82 \gamma^{2}-21 \gamma^{3}}{12(1-\gamma)^{3}}+O\left(B^{2}\right) \\
\quad \text { for } B \ll 1 \quad \text { and } \quad \gamma \leq O(1)
\end{gathered}
$$

This is an explicit result for the maximal critical diffusion $D_{\max }$ in the limit of small death rate $B$.

For buoyant phytoplankton with $\gamma<0$ and large $|\gamma|$, another approximation for the upper critical diffusion $D_{\max }$ can be derived from (72):

$$
\begin{aligned}
& \frac{D_{\max }}{A_{0}}=|\gamma| \frac{1-B}{B}+\frac{(1-B)^{3}}{B(1-B / 2)}+O\left(\frac{1}{\gamma}\right) \\
& \quad \text { for } \quad \gamma<0 \quad \text { and }|\gamma| \gg \max [4(1-B), B /(1-B)] .(95)
\end{aligned}
$$


For weakly sinking plankton with $\gamma>0$ and $\gamma \ll 2 \sqrt{1-B}$, the minimal critical diffusion $D_{\min }$ is, according to (75),

$$
\frac{D_{\min }}{A_{0}}=\frac{\gamma^{2}}{4(1-B)}\left(1+O\left(\frac{\gamma}{(1-B)^{2}}\right)^{2 / 3}\right) \quad \text { for } 0<\gamma \ll(1-B)^{2} .
$$

This last approximation reproduces the result of Riley et al. (1949) and Shigesada and Okubo (1981) that $D=v^{2} /\left(4 g\left(I_{i n}\right)\right)+\cdots$ and restricts its validity to $\gamma \ll$ $(1-B)^{2}$.

\section{Summary AND CONCluSIONS}

8.1. Theoretical summary. We have analysed the critical conditions for phytoplankton bloom development. Our results are particularly relevant for bloom development in eutrophic waters, as the model assumes that phytoplankton growth is determined by light availability only, whereas all nutrients are assumed to be available in ample supply. What distinguishes our analysis from many previous analyses of light-limited phytoplankton dynamics (Kok, 1952; Sverdrup, 1953; Evers, 1991; Platt et al., 1991; Huisman, 1999; Huisman et al., 1999a) is that we have here specifically focussed on incomplete mixing of phytoplankton (Shigesada and Okubo, 1981; Ishii and Takagi, 1982; Totaro, 1989; Huisman et al., 1999b,c). This paper confirms the recent numerical discovery of Huisman et al. $(1999 \mathrm{~b}, \mathrm{c})$ of a critical threshold value for the vertical turbulent diffusion coefficient by means of rigorous mathematical analysis. Furthermore, the paper extends the investigation to sinking and buoyant phytoplankton. Using dimensional analysis, the physical parameters like incident light intensity, background turbidity, water-column depth, maximal growth rate of phytoplankton, and so on reduce to the four dimensionless parameters $(A, B, C, L)$ defined in equation (16). These four parameters establish scaling rules and similarity laws between different phytoplankton-water-lightsystems. A transformation from dimensionless parameters back to physical parameters allows a straightforward interpretation of the conditions for phytoplankton bloom development in terms of measurable species traits and environmental conditions.

The full four-dimensional parameter space of phytoplankton bloom development cannot be displayed in a single three-dimensional plot. Therefore two different projections onto three-dimensional parameter spaces are represented in Figs 2 and 3. In the different panels of Fig. 2, the maximal water-column depth $L^{*}(A, B, C)$ is shown as a function of $A$ and $C$, and the panels are distinguished by different fixed values of $B$. Figure 3 shows the surface in the three-dimensional parameter space $(A, B, C)$, where the maximal water-column depth diverges $L^{*}(A, B, C) \rightarrow \infty$. The water-column depth $L$ can be treated as being infinite, from the perspective of phytoplankton bloom development, if $L \gg 1 / v$ in dimensionless parameters, 
or if $H \gg D / \sqrt{4 \ell D+v^{2}}$ in physical parameters. Various analytical results and asymptotic expansions for the phase boundaries of phytoplankton bloom development, in terms of the dimensionless parameters, are derived in Section 6.

In Section 7, these results are transformed back to physical parameters, and the complete scenario of critical diffusion vs critical depth is visualized in Fig. 5, as well as evaluated analytically. We emphasize that the three different types of diagrams of critical depth vs critical diffusion in Fig. 5 are distinguished by two parameters only, namely $B$ and $\gamma$. The parameter $B=\ell /\left(a I_{i n}^{\alpha}\right)$ is the ratio between death and reproduction rate of the phytoplankton at the surface, and $\gamma=$ $v \alpha K_{b g} /\left(a I_{i n}^{\alpha}\right)$ is the ratio between sinking velocity times light absorption coefficient and the reproduction rate at the surface.

Our analysis was restricted to growth equations in the form of (6) and (9). This choice kept the dimensionless parameter space four-dimensional, and allowed our transformation to Bessel functions and the use of their tabulated properties. An analysis of more complex growth equations in the form of (7) or (8) would add an additional 10th physical parameter $c$, which would make the dimensionless parameter space five-dimensional. In that case, our four-dimensional results with $\alpha=1$ would apply to low light conditions only ( $I_{i n} \ll 1 / c$, to be precise), where the $g(I)$ function is still in its linear range. However, the nonlinear behaviour of $g(I)$ for larger $I$ can be mimicked by an exponent $0<\alpha<1$ in (9), and this form of $g(I)$ is fully covered by our analysis.

8.2. General summary and conclusions. From a biological perspective, we found that the conditions for bloom development depend quite sensitively on the specific weight and hence on the vertical velocity of the phytoplankton species concerned. Our results can be characterized as follows:

- Bloom conditions for positively buoyant phytoplankton and neutrally buoyant phytoplankton are summarized in Fig. 5(a). They can develop blooms in highly turbulent waters if the water-column depth is less than the critical depth (Sverdrup, 1953). They can develop blooms in waters with an intermediate or low turbulent diffusion independent of water-column depth;

- Bloom conditions for sinking phytoplankton with a low to moderate sinking velocity are summarized in Fig. 5(b). They can develop blooms in highly turbulent waters if the water-column depth is less than the critical depth. Also, they can develop blooms in quiet waters if the water-column depth is less than the compensation depth. Finally, in waters where turbulent diffusion has a value between the minimal and the maximal turbulent diffusion, they can develop blooms independent of water-column depth;

- Bloom conditions for sinking phytoplankton with a high sinking velocity are summarized in Fig. 5(c). They cannot develop blooms in deep waters. They can develop blooms in turbulent shallow waters if the water-column depth is less than the critical depth, and in quiet shallow waters if the water column depth is less than the compensation depth. 
Thus, compared to previous work that studied incomplete mixing of neutrally buoyant plankton (Huisman et al., 1999b,c), this paper shows that sinking phytoplankton species have either two or no critical threshold values for the vertical turbulent diffusion coefficient [Fig. 5(b) and 5(c)]. In contrast, positively buoyant and neutrally buoyant phytoplankton always have one critical threshold value for the vertical turbulent diffusion coefficient, since a minimal turbulent diffusion $D_{\min }$ does not exist for buoyant species [Fig. 5(a)].

Intuitively, these various patterns can be explained by the light requirements of phytoplankton and the position they can obtain in the vertical light gradient. Neither buoyant nor sinking phytoplankton species can persist if vigorous mixing exposes the phytoplankton population to the low depth-averaged light conditions of deep waters [upper right corner in Fig. 5(a)-5(c)]. Phytoplankton species with a low sinking velocity cannot persist in deep waters if turbulent diffusion is too low to prevent sinking losses of phytoplankton into the dark [lower right corner in Fig. 5(b) and 5(c)], but these phytoplankton species may persist if turbulent diffusion has intermediate values, because growth rates may then overcome both mixing rates and sinking losses. Phytoplankton species with a high sinking speed cannot persist in deep waters at all, independent of turbulence, because their growth rate is insufficient to compensate for the sinking losses [Fig. 5(c)].

These qualitative considerations together with depth profiles, with some $(D, L)$ plots of the type of Figs. 5(b) and 5(c) and with population size data between the phase boundaries can be found in Huisman et al. (2001 or 2002).

Many empirical studies confirm the importance of vertical mixing processes for phytoplankton bloom development (Reynolds et al., 1983; Jones and Gowen, 1990; Cloern, 1991; Berman and Shteinman, 1998). One interesting example of an indepth study supporting the theory comes from Lake Nieuwe Meer, a deep and eutrophic lake in The Netherlands (Visser et al., 1996a,b). In former days, the phytoplankton of Lake Nieuwe Meer was dominated by Microcystis, a buoyant cyanobacterial species that can form toxic algal blooms. Artificial increase of vertical turbulent diffusion in the lake, by means of large-scale air bubbling, led to the replacement of buoyant Microcystis by sinking phytoplankton species, especially several diatoms and the green alga Scenedesmus (Visser et al., 1996a). Consistent with these field observations, laboratory experiments with Scenedesmus showed that this sinking species is lost from the water column if turbulent diffusion is too low (Visser et al., 1996b). Hence, this in-depth study underscores the idea that an increased turbulent mixing of the water column may lead to shifts in species composition from buoyant species towards sinking phytoplankton species, in line with the theory developed here.

As a general message, our model analysis and the given empirical example illustrate that incomplete mixing has a major impact on phytoplankton dynamics. Although incorporation of mixing processes in plankton models is gradually becoming more popular, there are still many models in plankton ecology and oceanography that lack information on the turbulence structure of the water column. 
Numerous models, sometimes even used as policy tools in water management, simply assume uniform mixing of the phytoplankton populations within the upper water column. Our mathematical analysis suggests that such simplified model approaches, that neglect the turbulence structure of the water column, might seriously underestimate opportunities for phytoplankton bloom development.

\section{ACKNOWLEDGEMENTS}

We thank the referees for their helpful comments. UE was supported by the Netherlands Organization for Scientific Research NWO, and MA by a TMR-grant of the European Union. BS and JH were supported by the Earth and Life Sciences Foundation (ALW) of NWO.

\section{REFERENCES}

Abramowitz, M. and I. A. Stegun (1964). Handbook of Mathematical Functions with Formulas, Graphs and Mathematical Tables, Nat. Bur. Standards Appl. Series 55, Washington, D.C.: U.S. Government Printing Office (paperback edition published by Dover, New York).

Arrigo, K. R., D. H. Robinson, D. L. Worthen, R. B. Dunbar, G. R. DiTullio, M. VanWoert and M. P. Lizotte (1999). Phytoplankton community structure and the drawdown of nutrients and $\mathrm{CO}_{2}$ in the Southern Ocean. Science 283, 365-367.

Berman, T. and B. Shteinman (1998). Phytoplankton development and turbulent mixing in Lake Kinneret (1992-1996). J. Plankton Res. 20, 709-726.

Britton, N. F. and U. Timm (1993). Effects of competition and shading in planktonic communities. J. Math. Biol. 31, 655-673.

Cloern, J. E. (1991). Tidal stirring and phytoplankton bloom dynamics in an estuary. J. Mar. Res. 49, 203-221.

Denny, M. W. (1993). Air and Water: The Biology and Physics of Life's Media, Princeton: Princeton University Press.

DiTullio, G. R., J. M. Grebmeier, K. R. Arrigo, M. P. Lizotte, D. H. Robinson, A. Leventer, J. P. Barry, M. L. VanWoert and R. B. Dunbar (2000). Rapid and early export of Phaeocystis antarctica blooms in the Ross Sea, Antarctica. Nature 404, 595-598.

Evers, E. G. (1991). A model for light-limited continous cultures: growth, shading, and maintenance. Biotechnol. Bioeng. 38, 254-259.

Falkowski, P. G., R. T. Barber and V. Smetacek (1998). Biogeochemical controls and feedbacks on ocean primary production. Science $281,200-206$.

Huisman, J. (1999). Population dynamics of light-limited phytoplankton: microcosm experiments. Ecology 80, 202-210.

Huisman, J., M. Arrayás, U. Ebert and B. Sommeijer (2001 or 2002). How do sinking phytoplankton species manage to persist? Am. Naturalist [to appear]. 
Huisman, J., R. R. Jonker, C. Zonneveld and F. J. Weissing (1999a). Competition for light between phytoplankton species: experimental tests of mechanistic theory. Ecology $\mathbf{8 0}$, 211-222.

Huisman, J., P. van Oostveen and F. J. Weissing (1999b). Critical depth and critical turbulence: two different mechanisms for the development of phytoplankton blooms. Limnology and Oceanography 44, 1781-1788.

Huisman, J., P. van Oostveen and F. J. Weissing (1999c). Species dynamics in phytoplankton blooms: incomplete mixing and competition for light. Am. Naturalist 154, 46-68.

Huisman, J. and F. J. Weissing (1994). Light-limited growth and competition for light in well-mixed aquatic environments: an elementary model. Ecology 75, 507-520.

Ishii, H. and I. Takagi (1982). Global stability of stationary solutions to a nonlinear diffusion equation in phytoplankton dynamics. J. Math. Biol. 16, 1-24.

Jones, K. J. and R. J. Gowen (1990). Influence of stratification and irradiance regime on summer phytoplankton composition in coastal and shelf seas of the British Isles (UK). Estuarine Coastal and Shelf Science 30, 557-568.

Kok, B. (1952). On the efficiency of Chlorella growth. Acta Botanica Neerlandica 1, 445-467.

Landau, L. D. and E. M. Lifshitz (1966). Course of Theoretical Physics, Vol. 6: Fluid Dynamics, 3rd edn, Oxford: Pergamon.

Monod, J. (1950). La technique de culture continue, theorie et applications. Annales de l'Institut Pasteur (Paris) 79, 390-410.

Okubo, A. (1980). Diffusion and Ecological Problems: Mathematical Models, Berlin: Springer.

Platt, T., D. F. Bird and S. Sathyendranath (1991). Critical depth and marine primary production. Proceedings of the Royal Society of London B 246, 205-217.

Platt, T., C. L. Gallegos and W. G. Harrison (1980). Photoinhibition of photosynthesis in natural assemblages of marine phytoplankton. J. Mar: Res. 38, 687-701.

Reynolds, C. S. (1984). The Ecology of Freshwater Phytoplankton, Cambridge: Cambridge University Press.

Reynolds, C. S., S. W. Wiseman, B. M. Godfrey and C. Butterwick (1983). Some effects of artificial mixing on the dynamics of phytoplankton populations in large limnetic enclosures. J. Plankton Res. 5, 203-234.

Riley, G. A., H. Stommel and D. F. Bumpus (1949). Quantitative ecology of the plankton of the western North Atlantic. Bull. Bingham Oceanographic Collection 12, 1-169.

Shigesada, N. and A. Okubo (1981). Analysis of the self-shading effect on algal vertical distribution in natural waters. J. Math. Biol. 12, 311-326.

Sverdrup, H. U. (1953). On conditions for the vernal blooming of phytoplankton. Journal du Conseil Permanent International pour l'Exploration de la Mer 18, 287-295.

Totaro, S. (1989). Mutual shading effect on algal distribution: a nonlinear problem. Nonlinear Anal. Theory Methods Appl. 13, 969-986.

Visser, P. M., B. W. Ibelings, B. van der Veer, J. Koedood and L. R. Mur (1996a). Artificial mixing prevents nuisance blooms of the cyanobacterium Microcystis in Lake Nieuwe Meer, The Netherlands. Freshwater Biol. 36, 435-450. 
Visser, P. M., L Massaut, J. Huisman and L. R. Mur (1996b). Sedimentation losses of Scenedesmus in relation to mixing depth. Archiv für Hydrobiologie 136, 289-308.

Webb, W. L., M. Newton and D. Starr (1974). Carbon dioxide exchange of Alnus rubra: a mathematical model. Oecologia 17, 281-291.

Weissing, F. J. and J. Huisman (1994). Growth and competition in a light gradient. J. Theor. Biol. 168, 323-336.

Received 12 March 2001 and accepted 26 July 2001 